\title{
ESTIMACIÓN DE LOS RETORNOS
} DE LA INVERSIÓN EN EDUCACIÓN SOBRE LOS SALARIOS DE LOS JEFES DE HOGAR EN COLOMBIA PARA EL AÑO 2018

\section{ESTIMATED RETURNS ON INVESTMENT IN EDUCATION ON THE WAGES OF HEADS OF}

\section{HOUSEHOLD IN COLOMBIA FOR 2018}

\author{
Héctor Javier Fuentes López', \\ Leonardo Emiro Contreras Bravo 2 \\ Karolina González Guerrero ${ }^{3}$
}

\section{RESUMEN}

Los retornos a la educación, medidos a través 1 Economista, Estudiante de ddoctorado en Economía. Docente de planta. Facultad de ingeniería. Universidad Distrital Francisco José de Caldas (Colombia). chifuentesl@udistrital.edu.co ORCID: https://orcid.org/00000001-6899-4564

2 Ingeniero, Estudiante de doctorado en ingeniería, Docente de planta. Facultad de ingeniería. Universidad Distrital Francisco José de Caldas (Colombia). lecontrerasb@ udistrital.edu.co. ORCID: https://orcid.org/0000-0003-4625$\underline{8835}$

3 Licenciada, Doctora en educación. Docente de planta. Facultad de estudios a distancia. Universidad Militar Nueva Granada. Karolina.gonzalez@unimilitar.edu.co kgonzalezg@gmail.com ORCID: https://orcid.org/0000-0002$\underline{9762-579 X}$ del salario, que perciben los trabajadores son una forma de medir que tan rentable resulta para un individuo su inversión tanto en tiempo como en recursos financieros. Los trabajadores asumen un costo de oportunidad cuando deciden dedicar su tiempo a mejorar sus habilidades y esperar mejores retornos medidos en su salario devengado. El presente artículo busca evidencia que permita corroborar qué tan rentable resulta, para un trabajador colombiano, los recursos dedicados a su capacitación. A partir de la teoría del capital humano, se construye un modelo que 
estima dichos retornos junto con otros elementos que definen el salario promedio de un trabajador en Colombia

\section{PALABRAS CLAVE}

Capital humano, retornos a la educación, modelos de regresión, salarios, jefes de hogar.

\section{ABSTRACT}

Returns to education, measured through the wages received by workers, are a way of measuring how profitable it is for an individual to invest both in time and financial resources. Workers bear an opportunity cost when they decide to spend their time improving their skills and expecting better returns measured in their earned wages. This article seeks evidence to corroborate how profitable, for a Colombian worker, the resources dedicated to their training. From the theory of human capital, a model is constructed that estimates these returns along with other elements that specify the average salary of a Colombian worker.

\section{KEYWORDS}

Human capital, returns to education, regression models, wages, heads of household.

\section{INTRODUCCIÓN}

Los estudios que evalúan la relación entre salarios y educación son abundantes y ha sido un tema ampliamente estudiado. Las inversiones tanto en tiempo como en recursos económicos asumidos suponen retornos futuros que se miden a partir de la rentabilidad que representa un mayor nivel salarial que compensa el esfuerzo y el riesgo tomado. Existe igualmente consenso en que los individuos más educados no solo perciben mejores ingresos laborales, sino que presentan tasas de desempleo menor y tienen mejores y mayores oportunidades de ascenso y desarrollo personal. Desde una perspectiva teórica, J. Mincer (1974) fue quien propuso una ecuación matemática para medir dichos retornos, pero los postulados teóricos se remontan a las aportaciones dadas por Adam Smith en el siglo XVIII, quien comparaba la inversión en educación con una maquinaria costosa y cuyos los retornos dependerán del gasto asumido por el individuo para mejorar su capacitación. Las inversiones en educación igualmente son analizadas desde la óptica del capital humano, es decir, el nivel de productividad en términos de las habilidades y de la experiencia adquirida, que igualmente, se compensan a través de las mejoras salariales que surgen de esta mayor productividad.

En este sentido, el objetivo principal de este trabajo es conocer desde una óptica cuantitativa la relación entre salarios y educación para Colombia, a partir de datos de corte transversal tomados de la encuesta de calidad de vida (ENCV) durante el año 2018, con el propósito de tener evidencia de cuanto es el aporte de los cambios salariales que obedecen a una mejora en educación. Para lograr este propósito se ha dividido el trabajo en seis partes incluyendo esta introducción. En la segunda sección se describen los elementos teóricos que soportan esta discusión en la que se expone tanto la teoría del capital humano como las aproximaciones mincerianas que metodológicamente soportan los cálculos respectivos. En la tercera sección, se describen los elementos metodológicos, que describen los datos y el modelo a estimar. La cuarta y quinta parte de este trabajo, muestra los resultados y la discusión de los mismos, en donde se presentan los cálculos numéricos que validan su relevancia teórica y permiten destacar ciertos análisis de la relación entre las variables utilizadas. Por último, se adjuntan las conclusiones obtenidas de la investigación junto con la bibliografía consultada y citada en el documento.

\section{REVISIÓN DE LA LITERATURA}

Smith (1776) fue uno de los pioneros en reconocer 
que el salario aumenta debido a la recuperación de la inversión hecha para alcanzar calificación en una labor y la estabilidad laboral que genera, entre otras ventajas adicionales (Cuevas, 1996). Igualmente, el trabajo de Cannan (1928), afirma que se si se usara el modelo de competencia perfecta, entonces habría equilibrio en los salarios independientemente de la profesión que se tenga, así que investigando respecto al porqué se daba dicha diferencia, encontró que uno de los factores más taxativo es el nivel educativo de preparación del trabajador o su habilidad respecto a la labor realizada (Suarez, 2018).

Walsh (1965), hace una comparación entre los ingresos promedio y el gasto en capital humano, es decir, en gastos relacionados con educación y formación para el trabajo y Solow (1957), incorpora el factor tecnológico dentro del modelo clásico de crecimiento, el cual define como todo aquello que aporta tanto al crecimiento de capital como de trabajo, haciendo precisiones significativas respecto a que la causa principal de dicho crecimiento está cimentada en los conocimientos que adquieren los individuos en su formación académica y profesional.

Por su parte, Schultz da formalidad a la teoría de capital humano, definiéndolo puntualmente como la equivalencia de hablar entre educación y formación. Además, según Falgueras (2008), Schultz defiende el uso del término sustentado en que el recurso humano es una forma de capital y por lo tanto el trabajo se debe ver como una ejecución manual, que está fundamentada en una habilidad y los conocimientos adquiridos; sin embargo, aparecen varios contradictores de Schultz, quienes plantean que no existe una diferencia entre los gastos de consumo y los gastos destinados a la inversión, con lo cual se debería ver el término capital humano como capital de trabajo, sin embargo, este autor no comparte esta posición y aunque si visualiza que hay poca claridad en la diferencia entre los tipos de gastos ya citados, afirma que la diferencia radica en distinguir entre los 5 grupos de actividades que mejoran el capital humano, dichos grupos son: inversión en salud y servicios, entrenamiento en el trabajo, educación básica primaria y secundaria, programas de estudios para adultos, migración de personas en busca de nuevas oportunidades (Suarez, 2018).

En Becker (1964) se define el capital humano como la suma de todas las capacidades que logran los individuos a de nuevos conocimientos, con el ideal de recibir salarios más significativos, es en Becker donde se describe a la inversión educación como el componente fundamental del capital humano, aunque como lo comprueba Mincer (1974), dicho capital no sólo está impulsado por la educación, sino por la experiencia adquirida por el trabajador, donde se concluye que el capital humano es determinante sobre la inversión social, además que la rentabilidad de la inversión en educación es evaluada sobre valores medibles, ya que las externalidades y rendimientos no monetarios son subjetivos según quien los evalué (Blaug 1983)

Cabe resaltar, por último, que otros trabajos recientes establecen claras relaciones entre capital humano, generación de ingresos y crecimiento económico; tales como Lucas (1988), Echart y Samuel (2001) y Virgüez, J. F., Sánchez Pineda, D. C., \& Rodríguez Suancha, S. A. (2020), quienes sustentan que el capital humano genera rendimientos crecientes que resaltan las diferencias entre el crecimiento económico de los países. Por su parte, Amartya Sen en 1999 aporta a la teoría haciendo énfasis en que esta no solo está constituida por factores como la educación, sino que además de esta se hacen participes la salud, a lo anterior y como trabajo complementario al de Sen ésta el de Bustamante (Sen y Bustamante citados en Cardona et al., 2007), quien en su trabajo del año 2003 afirma que además de la educación, 
salud, experiencia, el capital humano también lo conforman la seguridad, la cultura, entre otros. Otro aporte significativo es el de (Barro, 2013) con su trabajo en el año 2013, quien clasifica el Capital Humano como un factor alternativo al crecimiento económico, y enmarca que si bien viene cierto a este lo conforman factores como la salud y el componente de capital social, el factor más relevante es la educación (Suarez 2018).

\section{METODOLOGÍA}

A partir de los datos de la encuesta de calidad de vida medida por el Departamento Administrativo Nacional de Estadística para el año 2018, se tomaron datos de corte transversal para las características y composición del hogar en la que se incluyen variables de carácter social, económico y locacionales. Estos datos están tomados de una encuesta que considera 204876 individuos. A esta encuesta se le realiza un filtro para jefes de hogar que recibieron un salario monetario en este año y que fue al menos de un salario mínimo. Este proceso se realizó porque existen reportes de salarios muy bajos que denotan atipicidad y que no son el objetivo del presente análisis. Luego de realizar los filtros respectivos se encontró que de la encuesta presentada se obtuvo una submuestra de 7123 observaciones que cumplen con la restricción planteada.

De esta muestra se estima un modelo de regresión lineal múltiple que combina datos cualitativos como sexo, educación por niveles, estado civil con variables cuantitativas como salario, experiencia., edad y tenencia del empleo actual.

Se propone estimar una ecuación de salarios para jefes de hogar a partir de sus determinantes educacionales (primaria, secundaria, educación técnica, tecnológica, universitaria y de posgrado), junto con variables de edad, sexo, estado civil y experiencia laboral.
La ecuación anterior, representa la estructura general del modelo de regresión múltiple donde:

- Salario representa: salario de los jefes de hogar en Colombia en pesos colombianos.

- Exp representa los meses de experiencia laboral de los jefes de hogar en Colombia.

- Exp al cuadrado, se utiliza con el propósito de verificar el crecimiento decreciente de los salarios.

- Tenencia: variable cuantitativa que mide el número de meses que el jefe de hogar tiene con su empleo actual.

- Hombre: variable dicótoma donde uno es hombre y cero para mujer.

- Soltero: estado civil donde uno se le asigna a jefes de hogar soltero y cero para otro estado civil.

- Posgrado: variable dicótoma que toma valores de uno si la persona tiene posgrado y cero si no es el caso.

- Universitaria: variable dicótoma que toma valores de uno si la persona tiene título universitario y cero si no es el caso.

- Tecnología: variable dicótoma que toma valores de uno si la persona tiene título tecnológico y cero si no es el caso.

- Técnica: variable dicótoma que toma valores de uno si la persona tiene título técnico y cero si no es el caso.

- Primaria: variable dicótoma que toma valores de uno si la persona tiene educación primaria y cero si no es el caso. 
- Media: variable dicótoma que toma valores de uno si la persona tiene educación media y cero si no es el caso.

Para la estimación de la ecuación anterior se hizo con la metodología econométrica clásica para una ecuación de regresión múltiple. El proceso consistió en la organización de la base de datos, discriminación para jefes de hogar que tienen un salario igual o mayor a un sueldo mínimo, se transformaron las variables cualitativas en dicótomas y se obtuvieron los resultados a través del procedimiento de mínimos cuadrado ordinarios para los datos en niveles y el logaritmo natural del salario, con el objetivo de estimar las semi-elasticidades de las variables consideradas en el proceso.

\section{RESULTADOS}

Inicialmente se hace una descripción de las variables utilizadas en la estimación de la ecuación del modelo. Como se muestra en las tablas 1 y 2 de los datos seleccionados de la ENCV para el 2018, los jefes de hogar que recibieron ingreso de un salario mínimo o superior tienen una media de 992557 pesos colombianos para el año 2018. La edad promedio fue de 36.6 años y en promedio tenían 16 meses aproximadamente con el empleo actual, así como una experiencia laboral promedio de 27.6 meses

\section{ESTADÍSTICAS DESCRIPTIVAS}

Tabla 1.: Estadísticas descriptivas variables cuantitativas

\begin{tabular}{|cccccc|}
\hline Variable & Media & desviación & Asimetría & kurtosis & Coef var \\
Salarios & 992557 & 365732.4 & 2.209 & 13.45 & 0.460 \\
Edad & 36.6 & 11.966 & 0.666 & 3.079 & 0.327 \\
Tenencia & 15.7 & 25.03 & 2.813 & 9.468 & 1.595 \\
Experiencia & 27.6 & 49.84 & 3.684 & 20.20 & 1.807 \\
\hline
\end{tabular}

Fuente: autores a partir de la ENCV 2018

De la muestra considerada en su mayoría los jefes de hogar fueron hombres $(55 \%)$, tan solo el $27 \%$ era soltero, y en términos educativos el $45.5 \%$ tiene educación media o secundaria, el $18.5 \%$ educación primaria, el $21.5 \%$ posee educación técnica o tecnológica, y el $12 \%$ título universitario o de posgrado, a su vez tan solo el $2.3 \%$ no tiene ningún tipo de educación. 
Tabla 2.: Estadísticas descriptivas variables cualitativas

\begin{tabular}{|cccc|}
\hline Variable & sum & desviación & cv \\
& & & \\
Hombre & 3905 & 0.498 & 0.908 \\
Soltero & 1918 & 0.444 & 1.647 \\
Sin educación & 169 & 0.152 & 6.415 \\
Primaria & 1283 & 0.384 & 2.134 \\
Secundaria & 831 & 0.318 & 2.786 \\
Media & 2340 & 0.470 & 1.430 \\
Técnico & 1090 & 0.360 & 2.353 \\
Tecnológica & 390 & 0.225 & 4.213 \\
Universidad & 742 & 0.306 & 2.933 \\
Posgrado & 86 & 0.109 & 9.046 \\
\hline
\end{tabular}

Fuente: autores a partir de la ENCV 2018

La estimación del primer modelo, consideró el título máximo obtenido sin especificar si había algún tipo de educación incompleta. En este caso y como se muestra en la tabla 3 , se encontró que todas las variables son significativas al $5 \%$. Por medio de una estimación de mínimos cuadrados ordinarios con errores robustos se solucionaron los problemas de heteroscedasticidad propios de la heterogeneidad del tipo de datos. Se encuentra que los intervalos deducidos al $95 \%$ son adecuados y los signos esperados de los coeficientes igualmente son los correctos.

Tabla 3.: Resultados modelo 1

\begin{tabular}{|l|r|r|r|l|r|r|}
\hline & & Robust & & & & \\
\hline Logsalario & Coef. & Std. Err. & $\mathbf{t}$ & P>t & [95\% Conf. & Interval] \\
\hline & & & & & & \\
\hline Edad & 0.001 & 0.00 & 1.6 & 0.108 & 0.00 & 0.00 \\
\hline Experiencia & 0.003 & 0.00 & 13.8 & 0.000 & 0.00 & 0.00 \\
\hline Tenencia & -0.001 & 0.00 & -3.3 & 0.001 & 0.00 & 0.00 \\
\hline Exper2 & 0.000 & 0.00 & -9.1 & 0.000 & 0.00 & 0.00 \\
\hline Hombre & 0.236 & 0.01 & 22.7 & 0.000 & 0.22 & 0.26 \\
\hline Soltero & -0.084 & 0.01 & -7.7 & 0.000 & -0.10 & -0.06 \\
\hline Posgrado & 1.798 & 0.05 & 36.1 & 0.000 & 1.70 & 1.90 \\
\hline Universitario & 1.002 & 0.05 & 20.8 & 0.000 & 0.91 & 1.10 \\
\hline Tecnológico & 0.715 & 0.05 & 14.6 & 0.000 & 0.62 & 0.81 \\
\hline Técnico & 0.632 & 0.05 & 13.1 & 0.000 & 0.54 & 0.73 \\
\hline Media & 0.449 & 0.05 & 9.5 & 0.000 & 0.36 & 0.54 \\
\hline Secundaria & 0.264 & 0.05 & 5.4 & 0.000 & 0.17 & 0.36 \\
\hline Primaria & 0.122 & 0.05 & 2.5 & 0.012 & 0.03 & 0.22 \\
\hline Otro & 0.566 & 0.05 & 10.9 & 0.000 & 0.46 & 0.67 \\
\hline Constante & 12.92 & 0.05 & 240.6 & 0.000 & 12.82 & 13.03 \\
\hline
\end{tabular}

Fuente: autores a partir de la ENCV 2018 
Con el objetivo de considerar elementos adicionales con respecto a la categoría educación, se estimó un segundo modelo que incluyó variables de la encuesta que no se tuvieron en cuenta en una primera instancia. Aquí se incluyeron variables dicótomas para capturar el efecto de niveles de educación no terminado tales como estudios superiores no terminados. Así, la nueva estimación incluyó educación de posgrado, universitaria, técnica y tecnológica incompleta, tal y como se muestra en la tabla 4. La estimación, igualmente, se realizó a través de mínimos cuadrados ordinarios con errores robustos para solucionar problemas de heteroscedasticidad mencionados anteriormente. Los intervalos también deducidos al $95 \%$ son adecuados y los signos esperados de los coeficientes igualmente son los correctos.

Tabla 4.: Resultados modelo 2

\begin{tabular}{|l|r|r|r|r|r|r|}
\hline Logsalario & Coef. & Std. Err. & t & P>t & [95\% Conf. & Interval] \\
\hline & & & & & & \\
\hline Edad & 0.001 & 0.000 & 1.6 & 0.11 & 0.00 & 0.00 \\
\hline Experiencia & 0.002 & 0.000 & 13.7 & 0.00 & 0.00 & 0.00 \\
\hline Tenencia & -0.001 & 0.000 & -3.2 & 0.00 & 0.00 & 0.00 \\
\hline Exper2 & 0.000 & 0.000 & -9.0 & 0.00 & 0.00 & 0.00 \\
\hline Hombre & 0.237 & 0.010 & 22.8 & 0.00 & 0.22 & 0.26 \\
\hline Soltero & -0.083 & 0.011 & -7.6 & 0.00 & -0.10 & -0.06 \\
\hline Posgrado & 1.799 & 0.050 & 36.1 & 0.00 & 1.70 & 1.90 \\
\hline Posgradoincompleto & 1.555 & 0.106 & 14.7 & 0.00 & 1.35 & 1.76 \\
\hline Universitario & 1.002 & 0.048 & 20.8 & 0.00 & 0.91 & 1.10 \\
\hline Universidadincompleta & 0.586 & 0.054 & 10.8 & 0.00 & 0.48 & 0.69 \\
\hline Tecnológico & 0.715 & 0.049 & 14.6 & 0.00 & 0.62 & 0.81 \\
\hline Tecnológicoincompleto & 0.510 & 0.070 & 7.3 & 0.00 & 0.37 & 0.65 \\
\hline Técnico & 0.632 & 0.048 & 13.1 & 0.00 & 0.54 & 0.73 \\
\hline Técnicoincompleto & 0.524 & 0.059 & 8.8 & 0.00 & 0.41 & 0.64 \\
\hline Media & 0.449 & 0.047 & 9.5 & 0.00 & 0.36 & 0.54 \\
\hline Secundaria & 0.264 & 0.049 & 5.4 & 0.00 & 0.17 & 0.36 \\
\hline Primaria & 0.122 & 0.049 & 2.5 & 0.01 & 0.03 & 0.22 \\
\hline Constante & 12.92 & 0.054 & 240.5 & 0.00 & 12.82 & 13.03 \\
\hline
\end{tabular}

Fuente: autores a partir de la ENCV 2018 


\section{INTERPRETACIÓN Y DISCUSIÓN DE RESULTADOS}

De la estimación mostrada en la tabla 3 se puede ver que aumentos en la edad disminuyen el salario. Un incremento en el salario de un año viene acompañado de una disminución en el salario promedio de los jefes de hogar en Colombia del $0.1 \%$. En el caso de la experiencia se encuentra una relación positiva y en consecuencia, un mes más de experiencia tiene un efecto positivo del $0.3 \%$ en los salarios. Un mes adicional con el mismo empleo tiene un efecto inverso y afecta al salario en menos $0.1 \%$. Se encuentra que ser hombre en promedio incrementa el salario en $23 \%$ comparativamente con las mujeres, esto muestra un efecto discriminatorio a favor de los hombres y en contra de las mujeres. En términos de estado civil, en promedio, las personas solteras tienen un salario del $8 \%$ menor que el de las personas que tienen un estado civil diferente como casados, unión libre, viudo o divorciado. Este es un valor lógico si se tienen en cuenta que las personas solteras no tienen tantos gastos asociados al mantenimiento y crianza de una familia.

Por otra parte, y centrándose en el objetivo principal del trabajo, para el efecto educativo sobre el salario de los jefes de hogar en Colombia, se encontró, que en promedio y tomando como base, no tener ningún tipo de educación, una persona con educación primaria tiene un $12 \%$ más de salario, bachiller tiene en promedio un $26 \%$ más de salario, en tanto que un técnico tiene un $63 \%$ de mayor salario y una persona con educación tecnológica un $71.5 \%$. Para educación universitaria y posgradual los valores obtenidos son de un $100 \%$ y del $180 \%$ respectivamente. Es decir, en el caso de la educación universitaria en promedio representa el doble del salario y de la de posgrado es casi tres veces mayor.
Al analizar dentro del modelo los tipos de educación incompleta mostrados en la tabla 4 se encontraron valores similares para las variables antes consideradas, pero adicionalmente se muestra que tener algún tipo de educación así sea incompleta, afecta positivamente los salarios. En el caso de la educación técnica incompleta, su efecto sobre el salario, comparadas con aquellas que no tiene educación, se encontró que el salario puede ser un $52 \%$ mayor para el caso de los estudios técnicos y tecnológicos no finalizados, un $59 \%$ para el caso de los estudios universitarios no terminados, y del $155 \%$ para los de posgrados aún sin terminar.

Por último, los modelos estimados son coherentes con los resultados de los estudios y modelos mincerianos estimados para el caso de Colombia. En todos los caos se resalta como la educación genera aportes sustanciales al salario y como a mediada que aumentan los niéveles igualmente aumenta el salario incluso para los casos donde no se cuenta con un título de educación superior, pero si con estudios sin finalización. Tener un salario mayor depende del nivel de formación del jefe del hogar y su efecto crece en la medida que mayor sea el nivel de escolaridad, lo anterior, a la vez es consecuente con trabajos como: Isaza, J (2013), Hernández, G (2010), Urciaga, J (2002), Psacharopoulos, G., \& Chu Ng, Y (1992) y Kugler, de Gómez \& Posada. (1979), en los cuales se concluye que la formación académica si es diferencial sobre los salarios, que hay brechas significativas entre niveles de escolaridad secundaria y las de educación superior, y que además la tasa de retorno de la educación primaria es la más baja (Suarez, 2018).

\section{CONCLUSIONES}

Las brechas salariales en Colombia dependen de muchos determinantes, pero se destacan el género y la educación como principales determinantes de los ingresos de los jefes de hogar. Sobresale como la educación impacta de 
manera significativa especialmente la educación universitaria y posgradual que permite que los jefes de hogar doblen o incluso tripliquen sus ingresos. Mostrando esto un patrón de retornos a la educación de alta rentabilidad. Por lo anterior, se hace necesaria la profundización y el mantenimiento de la política pública que profundice las oportunidades de acceso a la educación superior, pues esta muestra ser el instrumento por excelencia para eliminar no solo las brechas de ingresos, sino una de las pocas salidas para abandonar la pobreza.

Aunque no todos tienen la oportunidad de realizar un posgrado, si se encuentran importantes retornos a la inversión realizada en este ámbito, incrementando sustancialmente sus retornos y casi doblando la inversión realizada en estudios universitarios de pregrado. Junto con una combinación de experiencia y estudios posgraduales los trabajadores no solo mejoran su capital humano, sino que esta mejora compensa sustancialmente los esfuerzos de tiempo y dinero invertido.

No deja de ser llamativo la discriminación de género en el país, que en promedio representa $23 \%$ y que abre la posibilidad para estudiar a mayor profundidad este fenómeno, si se discrimina género por nivel educativo y por región muy seguramente se encontrarán resultados más desagregados dado que el país no solo muestra brechas de género, sino que además el tema regional muestra brechas sustanciales e igualmente este es otro desafío de la política pública en el país.

\section{REFERENCIAS BIBLIOGRÁFICAS}

Cardona, M., Montes, I., Vasquez, J., Villegas, M., \& Brito, T. (2007). Capital humano. Una mirada desde la educación y la experiencia laboral. Cuadernos de Investigación, (56).

Briceño, A. (2010). La educación y su efecto en la formación de capital humano y el desarrollo económico de los países. Apuntes del CENES. Vol.30 (51), pp. 45-59.

Barro, R. (2013). Education and Economic Growth. Annals of Economics and Finance. Vol. 12 (2 A), pp. 277-304.

Becker, G. 1964. Human Capital: A Theoretical and Empirical Analysis with Special Reference to Education. New York: NBER Columbia University Press.

Cannan, Edwin: Wealth. A Brief Explanation of the Causes of Economic Welfare, London: P.s. King and son, 1928 (3rd. ed.).

Cuevas, H. (1996). El Capital Humano en el sistema de precios. Cuadernos de Economía. pp. 9-25.

Denison, E. (1962). "The Sources of Economic Growth in the United States and the Alternatives Before us". Committee of Economic Development.

Falgueras, I. (2008). La Teoría del Capital Humano: Orígenes y Evolución. Temas actuales de economía. Universidad de Málaga. Recuperado de: http://www. economiaandaluza.es/sites/default/ files/La\%20teor\%C3\%ADa\%20del\%20 capital\%20humano

Garrido, C. (2007). La educación desde la teoría del capital humano y el otro. Educare. Vol.11 (36), pp. 73-80.

Gujarati, D., \& Porter, D. (2010). Econometría (quinta edición). México: McGRAW-HILL/ INTERAMERICANA EDITORES, SS DE CV. pp. 541-542.

Hernández, G. (2010). ¿Cuán rentable es la educación superior en Colombia? Lecturas de Economía Nro. 73, pp.184-214. 
Isaza Castro, J. G. (2013). Occupational segregation, gender wage differences and trade reforms: empirical applications for urban Columbia (PhD Thesis). University of Sussex.

Kugler, B., de Gómez, M. I., \& Posada, A. R. (1979). Educación y mercado de trabajo urbano en Colombia: una comparación entre sectores modernos y no modernos. Corporación Centro Regional de Población, Area Socio-Económica.

Mincer, J. (1974). Schooling, Experience, and Earnings. Human Behavior \& Social Institutions No. 2.

OCDE. (1998). Measuring what people know: Human capital for the knowledge economy. Francia: OECD Publications. Recuperado de: http://www.oecd-ilibrary.org/docserver/download/9196031e. pdf?expires $=1517287473 \&$ id=id\&accname $=$ guest\&checksum $=$

\section{A3A674E85ABE89F4D70EED0E7B51A79B}

Psacharopoulos, G., \& Chu Ng, Y. (1994). Earnings and education in Latin America.

Education Economics, 2(2), 187-207.

Sen, Amartya. 1999. Development as freedom. Oxford: Oxford University Pres

Solís, F. \& Limas, M. (2012). Capital social y desarrollo: origen, definiciones y dimensiones de análisis. Recuperado de: http://www.redalyc.org/html/859/85927874008/.

Solow, Robert M.: "A Technical Change and the Aggregate Production Function", Review of Economics and Statistics, 39 (August), 1957, 312-20.

Suarez Diaz, Erika Alejandra. (2018). Análisis de los retornos de la educación de los jefes de hogar en Colombia (2013-2015), a partir de modelos probabilísticos. Tesis de Maestría en Economía. Uptc, Tunja.

Urciaga, J. (2002). Los rendimientos privados de la escolaridad formal en México. Revista Comercio Exterior, 52, 324-330.

Villalobos, G. \& Pedroza, R. (2009). Perspectiva de la teoría del capital humano acerca de la relación entre educación y desarrollo económico. Revista Internacional de investigación educativa. Vol.10 (20), pp. 273-306.

Virgüez, J. F., Sánchez Pineda, D. C., \& Rodríguez Suancha, S. A. (2020). La comunicación de marketing en el comportamiento del consumidor para las organizaciones: revisión sistemática de literatura. Revista Boletín Redipe, 9(1), 174-183. https://doi.org/10.36260/rbr.v9i1.906

Walsh, John R.: "Capital Concept Applied to man", Quarterly Journal of Economics, XLIX, 1935, 255285. 